\title{
Performance of fractional tap-spacing equalizers for long-haul high-capacity digital fibre-optic communication systems
}

\author{
F. CASADEVALL, J. PASTOR \\ ETS Ingenieros de Telecommunicaciòn, Universidad Politècnica de Catalunya, \\ PO 30002, 08034 Barcelona, Spain
}

Received 12 August 1985; revised 28 January; accepted 27 February 1986

It is known that for data transmission above $100 \mathrm{Mbits}^{-1}$ multimode fibre systems, working at $1.3 \mu \mathrm{m}$ wavelength, are more modal-dispersion-limited than loss-limited. Then some form of baseband equalization is highly desirable. Baseband equalizers are usually made in the form of a transverse filter with variable tap gains and tap spacing equal to the symbol spacing $T$. However, the performance of these equalizers depends critically on the timing phase. To overcome this difficulty, in this paper we analyse the performances of fractional tap-spacing equalizers that have a tap spacing smaller than $T$. Results obtained show that this type of equalizer gives satisfactory performance for a broad continuous range of timing phase values. It allows the application of simple clock recovery schemes in the receiver.

\section{Introduction}

It is well known that by using multimode optical fibres, with wavelengths beyond approximately $1.3 \mu \mathrm{m}$, the repeater spacing is limited by the modal dispersion at data rates above $100 \mathrm{Mbits} \mathrm{s}^{-1}$. When the repeater spacing increases, this dispersion degrades the equivalent baseband frequency response of the optical channel and produces an important increase of intersymbol interference (ISI). This ISI does not allow a benefit from weak attenuation of the optical fibre which can be lower than $1 \mathrm{~dB} \mathrm{~km}^{-1}$ at those wavelengths. Therefore, for long-haul systems it is desirable to use equalization techniques through transverse structures that mitigate the effects of ISI.

Different strategies for optimization of equalizer coefficients have been proposed up to now [1-4]. In all of these cases, the coefficient values of the equalizers and their performances have been obtained considering that the sampling instant, $t_{0}$, is in some sense optimum. However, the performance of these equalizers depends critically on the chosen sampling instant. Hence, in conjunction with the equalizer a clock recovery scheme must be used which provides a good clock phase.

To find an optimal sampling instant, several stochastic algorithms have been proposed [5-6]. However, in the real systems an open-loop clock recovery scheme is preferred. Today, the usual clock recovery scheme is comprised of rectifier or square-law characteristic circuit and a further phase-locked loop (PLL) which extracts the already generated spectral line at symbol rate $T^{-1} \mathrm{~Hz}$ [7]. In general, the sampling instant obtained can be different from the one considered in the equalizer calculation. In this case an increase in the received optical power is needed and, suddenly, the performance of the system drops quickly [2].

In this paper, we have investigated the possibility of eliminating the need for precise clock-phase control by choosing an equalizer with tap spacing somewhat smaller than the symbol spacing $T$. This equalizer is called a fractional tap-spacing equalizer (FTSE) [8]. We have only analysed linear equalizers because their performances are more sensitive to the timing phase than the performances 
of non-linear equalizers [9]. The taps on transverse filters of equalizers were computed using a minimum mean-square error (m.m.s.e.) and we have specially taken into account the dependence of shot noise in the received optical power.

In Section 2, the digital transmission problem for fibre channels is briefly analysed. The typical structures for FSTE are reviewed in Section 3 and their relative merits are discussed. Section 4 is devoted to the analysis of the system performance. In particular, we have used for the system design the strategy proposed in [3]. Finally, in the last section several results of practical interest are reported.

\section{System model}

The model that we have chosen to characterize the digital transmission system is shown in Fig. 1. The source, with rate $1 / T$, generates a sequence of statistically independent symbols, $\left\{a_{n}\right\}$, taking values from the binary alphabet $\{0,1\}$. The signal at the line encoder output modulates the intensity of a laser diode and the output power is transmitted through a multimode optical fibre. Then, the transmitted optical signal is

$$
P_{\mathrm{T}}(t)=P \sum_{n=-\infty}^{\infty} a_{n} h_{1}(t-n T)
$$

where $h_{1}(t)$ defines the pulse shape and $P$ is the peak value of the optical power injected into the fibre.

The optical fibre acts like a linear power channel [10], and is completely characterized by its transfer function. So, the optical power at the filter output is

$$
P(t)=P_{0} \sum_{n=-\infty}^{\infty} a_{n} h_{r}(t-n T)
$$

where $P_{0}(\mathrm{~dB} \mathrm{~m})=P(\mathrm{dBm})-\alpha L, \alpha$ is the fibre attenuation in $\mathrm{dB} \mathrm{km}^{-1}, L$ is the distance between repeaters, $h_{\mathrm{r}}(t)=h_{1}(t) * h_{2}(t) * h_{3}(t)$ is the fibre-optic response, without taking into account its attenuation, and $*$ is the convolution parameter.

On the receiver side, the photodetector and amplifier deliver a signal which is proportional to the transmitted optical power and noise. The amplifier is followed by a low-pass filter in order to improve its output signal-to-noise ratio. The joint frequency response of both elements have been denoted $H_{3}(f)$. The filter output signal is

$$
x(t)=S M P_{0} a_{0} h(t)+Z(t)+S(t)+n_{\mathrm{T}}(t)
$$

where

$$
Z(t)=S M P_{0} \sum_{\substack{n=-\infty \\ n \neq 0}}^{\infty} a_{n} h(t-n T)=\text { ISI }
$$

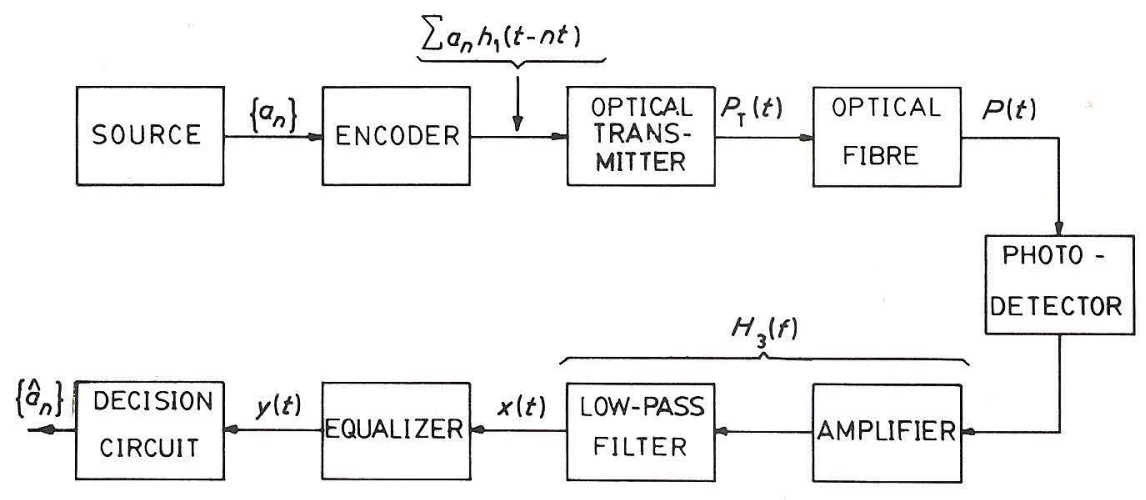

Figure 1 Block diagram of the optical system model showing the most important parameters. 
$S$ is the photodetector sensitivity $\left(\mathrm{AW}^{-1}\right), M$ is the avalanche gain of the photodetector, $h(t)=$ $h_{1}(t) * h_{2}(t) * h_{3}(t)$ is the system impulse response, $S(t)$ is the shot noise and $n_{\mathrm{T}}(t)$ is the thermal noise.

The shot noise is a non-stationary stochastic process which depends on the transmitted message and it is characterized by its moments [11].

To eliminate the ISI present in the output filter in Equation 3, we have used a fractionally spaced transverse equalizer.

\section{Fractional tap-spacing equalizer}

In general, a fractional tap-spacing equalizer has a structure as shown in Fig. 2. The delay line taps are spaced with an interval $\tau$, which is less than the symbol interval $T$. The tap spacing is usually selected so that $\tau$ is $K T / M$, where $K$ and $M$ are integers and $M>K$. The output signal $y(t)$ consists of the weighted sum of delayed versions of the input signal $x(t)$.

In practice, it is more convenient to choose $\tau=T / M$, where $M$ is a small integer. In our analysis we have only considered two samples in each interval $T$. Therefore, the structure of Fig. 2 can be represented as shown in Fig. 3 [12], where we have considered the FTSE as the linear combination of two conventional $T$-spaced equalizers whose input signals are delayed by $\tau$.

Hence, on a fibre-optic system the output signal, $y(t)$, is

$$
\begin{aligned}
y(t) & =\sum_{j=-K 1}^{K 2} C A_{j} x(t+j T)+\sum_{\substack{l=-K 3 \\
l \neq 0}}^{K 4} C B_{l} x(t+l T-\tau) \\
& =S M P_{0}\left[a_{0} h_{\mathrm{E}}(t)+\sum_{\substack{n=-\infty \\
n \neq 0}}^{\infty} a_{n} h_{\mathrm{E}}(t-n T)\right]+S_{\mathrm{E}}(t)+n_{\mathrm{E}}(t)
\end{aligned}
$$

where

$$
\begin{aligned}
& h_{\mathrm{E}}(t)=\sum_{j=-K 1}^{K 2} C A_{j} h(t+j T)+\sum_{\substack{l=-K 3 \\
l \neq 0}}^{K 4} C B_{l} h(t+l T-\tau) \\
& S_{\mathrm{E}}(t)=\sum_{j=-K 1}^{K 2} C A_{j} S(t+j T)+\sum_{\substack{l=-K 3 \\
l \neq 0}}^{K 4} C B_{l} S(t+l T-\tau) \\
& n_{\mathrm{E}}(t)=\sum_{j=-K 1}^{K 2} C A_{j} n_{\mathrm{T}}(t+j T)+\sum_{\substack{l=-K 3 \\
l \neq 0}}^{K 4} C B_{l} n_{\mathrm{T}}(t+l T-\tau)
\end{aligned}
$$

with $K 2$ and $K 1$ being, respectively, the number of leading and trailing taps on the upper $T$-spaced

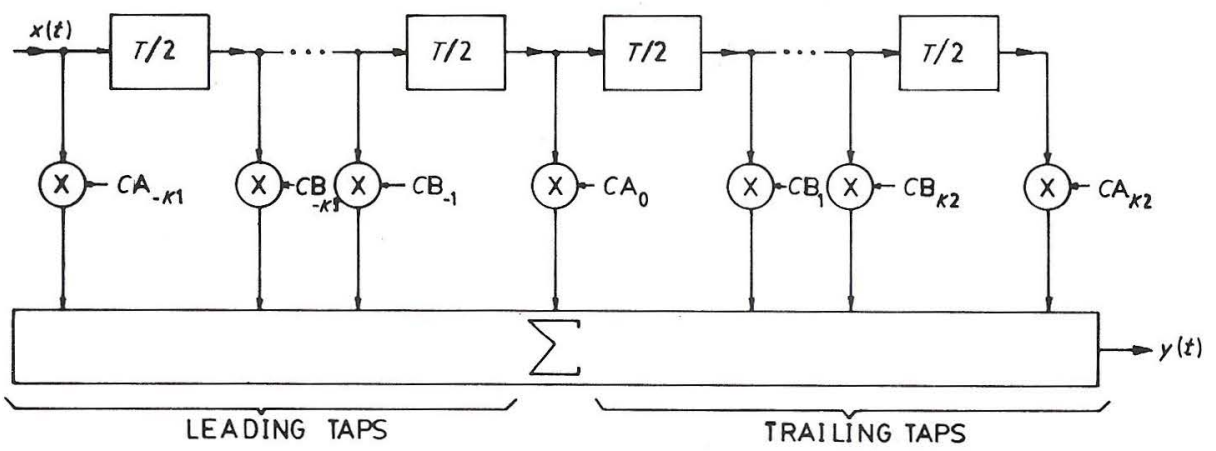

Figure 2 Fractional tap-space equalizer (FTSE). 


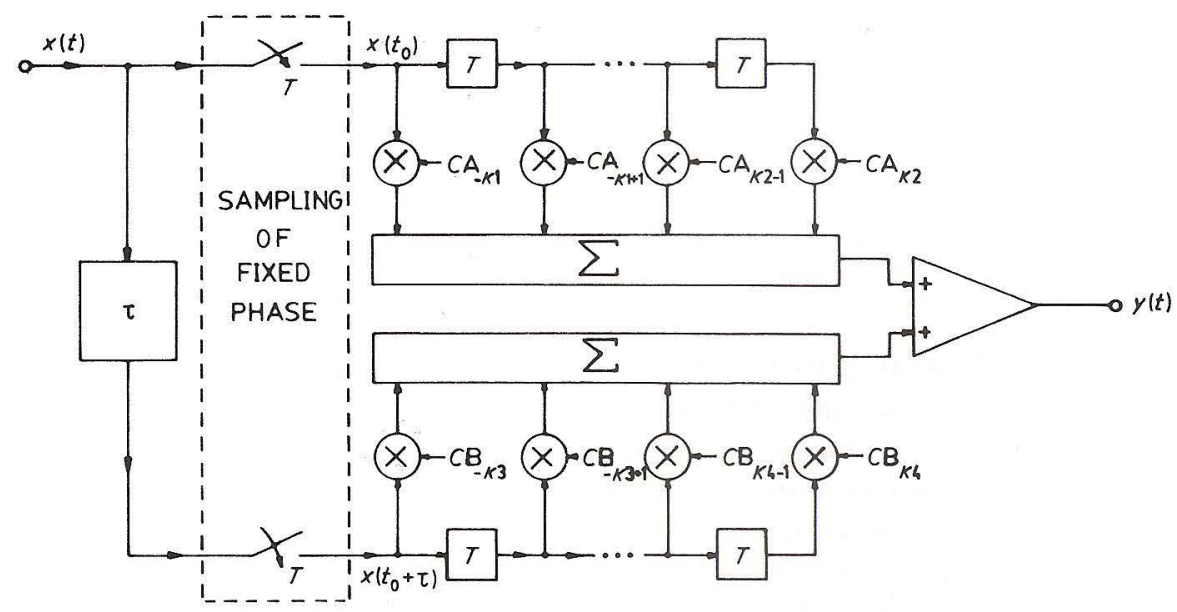

Figure 3 Equivalent scheme for fractional tap-space equalizer.

equalizer ( $K 4$ and $K 3$ being the corresponding quantities for the lower $T$-spaced equalizer). For an adequate operation of this structure as FTSE it is necessary to verify $K 1=K 3$ and $K 2=K 4$. In order to clarify the following explanation we will preserve the previous notation.

The number of taps on the upper part of the equalizer is $M A=K 1+K 2+1$, and the number of taps on the lower part is $M B=K 3+K 4$. Thus, the total number of taps in FTSE is $N T=M A+M B=2(K 1+K 2)+1$. Then, to compensate for the same amount of ISI, FTSE needs approximately twice as many taps as a conventional $T$-spacing equalizer.

The criterion chosen to compute the value of the equalizer tap consists in minimizing the mean square error, $\xi$, which is defined as the sampling time, $t_{0}$, as

$$
\xi=E\left\{\left|y_{l}-y_{l, \text { ideal }}\right|^{2}\right\}
$$

with $y_{l}=y\left(t_{0}+l T\right)$ and $y_{l, \text { idcal }}=S M P_{0} a_{l}$.

Without losing generality, we next assume that the symbol $a_{0}$ is sampled at the instant $t_{0}$.

The minimum value of the mean square error is obtained when

$$
\begin{aligned}
& \frac{\delta \xi}{\delta C A_{i}}= \frac{\delta}{\delta C A_{i}} E\left\{\mid\left[\sum_{j=-K 1}^{K 2} C A_{j} x\left(t_{0}+j T\right)\right.\right. \\
&\left.\left.+\sum_{\substack{l=-K 3 \\
l \neq 0}}^{K 4} C B_{l} x\left(t_{0}+l T-\tau\right)\right]-\left.S M P_{0} a_{0}\right|^{2}\right\}=0 \\
& \frac{\delta \xi}{\delta C B_{i}}= \frac{\delta}{\delta C B_{i}} E\left\{\mid\left[\sum_{j=-K 1}^{K 2} C A_{j} x\left(t_{0}+j T\right)\right.\right. \\
&\left.\left.+\sum_{\substack{l=-K 3 \\
l \neq 0}}^{K 4} C B_{l} x\left(t_{0}+l T-\tau\right)\right]-\left.S M P_{0} a_{0}\right|^{2}\right\}=0 \\
& i=-K 3, \ldots,-1,1, \ldots, K 4
\end{aligned}
$$

The above expression can be written as

$$
\begin{array}{cc}
\sum_{j=-K 1}^{K 2} C A_{j} E\left\{x\left(t_{0}+i T\right) x\left(t_{0}+j T\right)+\sum_{\substack{l=-K 3 \\
l \neq 0}}^{K 4} C B_{l} E\left\{x\left(t_{0}+i T\right) x\left(t_{0}+l T-\tau\right)\right\}\right. \\
=S M P_{0} E\left\{x\left(t_{0}+i T\right) a_{0}\right\} ; & i=-K 1, \ldots, 0, \ldots, K 2
\end{array}
$$




$$
\begin{gathered}
\sum_{j=-K 1}^{K 2} C A_{j} E\left\{x\left(t_{0}+j T\right) x\left(t_{0}+i T-\tau\right)\right\}+\sum_{\substack{l=-K 3 \\
l \neq 0}}^{K 4} C B_{l} E\left\{x\left(t_{0}+i T-\tau\right) x\left(t_{0}+l T-\tau\right)\right\} \\
=S M P_{0} E\left\{x\left(t_{0}+i t-\tau\right) a_{0}\right\} ;
\end{gathered}
$$

The form of Equation 11 can be simplified by using matrix notation. We can write

where $C$ is the vector of equalizer coefficients

$$
\tilde{A} C=S M P_{0} G
$$

$$
C=\left[C A_{-K 1}, \ldots, C A_{0}, \ldots, C A_{K 2}, C B_{-K 3}, \ldots, C B_{-1}, C B_{1}, \ldots, C B_{K 4}\right]^{\mathrm{T}}
$$

$\tilde{A}$ is the autocorrelation matrix of equalizer input signal, with a generic term $a_{n m}$

$$
a_{m n}= \begin{cases}R_{x x}\left[\left(t_{0}+i T\right),\left(t_{0}+j T\right)\right] ; & m=1, \ldots, M A \\ R_{x x}\left[\left(t_{0}+i T\right),\left(t_{0}+j T-\tau\right)\right] ; & i, j=-K 1, \ldots, 0, \ldots, K 2 \\ & m=1, \ldots, M A \\ & n=M A+1, \ldots, M A+M B \\ & i=-K 1, \ldots, 0, \ldots, K 2 \\ & j=-K 3, \ldots,-1,1, \ldots, K 4 \\ R_{x x}\left[\left(t_{0}+i T-\tau\right),\left(t_{0}+j T\right)\right] ; & m=M A+1, \ldots, M A+M B \\ & n=1, \ldots, M A \\ & i=-K 3, \ldots,-1,1, \ldots, K 4 \\ R_{x x}\left[\left(t_{0}+i T-\tau\right),\left(t_{0}+j T-\tau\right)\right] ; & m, n=-K 1, \ldots, 0, \ldots, K 3 \\ & i, j=-K 3, \ldots,-1,1, \ldots, K 4\end{cases}
$$

where $R_{x x}\left(t_{1}, t_{2}\right)=E\left\{x\left(t_{1}\right) x\left(t_{2}\right)\right\}$. $G$ is a vector of cross-correlation between the equalizer input signal and the transmitted symbol $\left\{a_{n}\right\}$. The generic term, $G_{i}$, of that vector is

where

$$
G_{n}= \begin{cases}R_{a x}\left[\left(t_{0}+i T\right), 0\right] ; & n=1, \ldots, M A \\ R_{a x}\left[\left(t_{0}+i T-\tau\right)\right] ; & n=M A+1, \ldots, M A+M B \\ i & =-K 3, \ldots,-1,1, \ldots, K 4\end{cases}
$$

$$
\begin{aligned}
R_{a x}\left(t_{1}, 0\right) & =E\left[x\left(t_{1}, a_{0}\right)\right] \\
& =E_{\left\{a_{n}\right\}}\left\{S M P_{0} \sum_{n=-\infty}^{\infty} a_{n} a_{0} h\left(t_{1}-n T\right)+S\left(t_{1}\right) a_{0}+n_{\mathrm{T}}\left(t_{1}\right) a_{0}\right\}
\end{aligned}
$$

The expression for $R_{x x}\left(t_{1}, t_{2}\right)$ is computed in the Appendix. By substituting $\lambda(t)=(S / q) P(t)+$ $I_{\mathrm{M}} / q$ in the Equation $\mathrm{A} 10$, we obtain

$$
\begin{aligned}
R_{x x}\left(t_{1}, t_{2}\right)= & E\left\{\left(S M P_{0}\right)^{2}\left[\sum_{j=-\infty}^{\infty} a_{j} h\left(t_{1}-j T\right)+I_{\mathrm{M}} H_{3}(0)\right]\left[\sum_{i=-\infty}^{\infty} a_{i} h\left(t_{2}-i T\right)+I_{\mathrm{M}} H_{3}(0)\right]\right\} \\
& +E\left[q M^{2} F(M) S P_{0} \sum_{j=-\infty}^{\infty} a_{j} h_{r}\left(t_{2}-j T\right) h_{3}\left(t_{1}-t_{2}\right) * h_{3}\left(t_{2}\right)\right] \\
& +q M^{2} F(M) I_{\mathrm{M}} h_{\mathrm{A}}\left(t_{1}-t_{2}\right)+R_{N N}\left(t_{1}, t_{2}\right)
\end{aligned}
$$


where $q$ is the electron charge, $I_{\mathrm{M}}$ is the photodetector multiplicative dark current

$$
\begin{aligned}
h_{\mathrm{A}}(t) & =h_{3}(t) * h_{3}(t) \\
F(M) & =K M+(1-K)\left(2-M^{-1}\right)
\end{aligned}
$$

$K$ is one parameter which depends on the material and doping profile of the avalanche diode, and $R_{N N}\left(t_{1}, t_{2}\right)$ is the autocorrelation function of $n_{\mathrm{T}}(t)$.

By considering $H_{3}(0)=0$, because the receiver has coupling capacitors, and

$$
E\left\{a_{i} a_{j}\right\}= \begin{cases}1 / 2 & i=j \\ 1 / 4 & i \neq j\end{cases}
$$

the expression of $R_{x x}\left(t_{1}, t_{2}\right)$ and $R_{a x}\left(t_{1}, n\right)$ results in

$$
\begin{aligned}
R_{x x}\left(t_{1}, t_{2}\right)= & \frac{1}{2}\left(S M P_{0}\right)^{2} R_{1}\left(t_{1}, t_{2}\right)+\frac{1}{2} S M^{2} F(M) P_{0} R_{2}\left(t_{1}, t_{2}\right) \\
& +R_{3}\left(t_{1}, t_{2}\right)+R_{N N}\left(t_{1}, t_{2}\right)
\end{aligned}
$$

and

$$
R_{a x}\left(t_{1}, n\right)=\frac{1}{2}\left(S M P_{0}\right)\left[h\left(t_{1}-n T\right)+\frac{1}{2} \sum_{\substack{l=-\infty \\ l \neq n}}^{\infty} h(t-l T)\right]
$$

where

$$
\begin{aligned}
R_{1}\left(t_{1}, t_{2}\right)= & \frac{1}{2} \sum_{\substack{i=-\infty \\
i \neq j}}^{\infty} \sum_{j=-\infty}^{\infty} h\left(t_{1}-i T\right) h\left(t_{2}-j T\right) \\
& +\sum_{i=-\infty}^{\infty} h\left(t_{1}-i T\right) h\left(t_{2}-i T\right) \\
R_{2}\left(t_{1}, t_{2}\right)= & q \sum_{j=-\infty}^{\infty}\left[h_{\mathrm{r}}\left(t_{2}-j T\right) h_{3}\left(t_{1}-t_{2}\right)\right] * h_{3}\left(t_{2}\right) \\
R_{3}\left(t_{1}, t_{2}\right)= & q M^{2} F(M) I_{\mathrm{M}} h_{\mathrm{A}}\left(t_{1}-t_{2}\right)
\end{aligned}
$$

Finally, the values of the tap equalizer are found by solving Equation 12 as

$$
C=S M P_{0} \tilde{A}^{-1} G
$$

\section{System performance}

The quality measuring parameter of a digital transmission system is the symbol error probability, $P_{\mathrm{e}}$.

In order to obtain the system performances we need to know the threshold value of received optical power that is necessary to guarantee that $P_{\mathrm{e}}=10^{-9}$. This threshold value depends on the system parameters $h(t), S, M, F(M)$ thermal and shot noise, etc., but mainly on the behaviour of FTSE. As the tap values of FTSE are also dependent on the threshold of received optical power it is necessary to resort to an iterative solution.

First, we obtain a bound in order to characterize the error probability. Then, the receiver optimization procedure is outlined.

\subsection{Error probability bound}

The calculation of the error probability in the presence of ISI and shot noise is very complex and so one resorts to obtaining bounds of that value. In [3] we had suggested an upper bound that can be favourably compared with previously published upper bounds. This upper bound can be 
expressed as (when $a_{0}=0$ )

$$
\begin{aligned}
P(\mathrm{e} / 0) & \leqslant \frac{1}{2} \operatorname{erfc}\left(\frac{D-F_{\infty}}{2^{1 / 2} \sigma_{S 0 M}}\right) \\
& \times \prod_{m=1}^{\infty}\left[\frac{1}{2}\left\{1+\frac{\operatorname{erfc}\left(\frac{D-F_{\infty}+\left|r_{m}\right|}{2^{1 / 2} \sigma_{S O M}}\right)}{\operatorname{erfc}\left(\frac{D-F_{\infty}}{2^{1 / 2} \sigma_{S O M}}\right)}\right\}\right]
\end{aligned}
$$

where $\left\{r_{i}\right\}$ is the succession of the interference samples arranged in absolute value, $D$ is the detector normalized threshold,

$$
F_{\infty}=\sum_{i=1}^{\infty} r_{i} u\left(r_{i}\right)
$$

$u($.$) is the step function$

$$
\begin{gathered}
\sigma_{S 0 M}^{2}=\left[q S M^{2} F(M) P_{0} \sum_{\substack{n=-\infty \\
n \neq 0}}^{\infty} h_{\mathrm{B}}\left(t_{0}-n T\right)+N+\sigma_{\mathrm{T}}^{2}\right] /\left(S M P_{0}\right)^{2} \\
h_{\mathrm{B}}(t)=h_{\mathrm{r}}(t) *\left[h_{3}(t) * h_{4}(t)\right]^{2} \\
N=2 q M^{2} F(M) I_{\mathrm{M}} B_{\mathrm{N}} \\
B_{\mathrm{N}}=\int_{0}^{\infty}\left[H_{3}(f) H_{4}(f)\right]^{2} \mathrm{~d} f
\end{gathered}
$$

$q$ is the electron charge, $F(M)$ is the excess noise factor due to the avalanche process, $h_{4}(t)$ is the equalizer impulse response and $I_{\mathrm{M}}$ is the photodetector multiplicative dark current.

When $a_{0}=1$

$$
\begin{aligned}
P(\mathrm{e} / 1) & \leqslant \frac{1}{2} \operatorname{erfc}\left(\frac{E_{\infty}-D}{2^{1 / 2} \sigma_{S 1 M}}\right) \\
& \times \prod_{m=1}^{\infty}\left[\frac{1}{2}\left\{1+\frac{\operatorname{erfc}\left(\frac{E_{\infty}-\left|r_{m}\right|+D}{2^{1 / 2} \sigma_{S 1 M}}\right)}{\operatorname{erfc}\left(\frac{E_{\infty}-D}{2^{1 / 2} \sigma_{S 1 M}}\right)}\right\}\right]
\end{aligned}
$$

where

$$
\begin{aligned}
E_{\infty} & =h_{\mathrm{E}}\left(t_{0}\right)+\sum_{i=1}^{\infty} r_{i}\left[1-u\left(r_{i}\right)\right] \\
\sigma_{S 1 M}^{2} & =\left[q S M^{2} F(M) P_{0} h_{\mathrm{B}}\left(t_{0}\right)+\sigma_{S 0 M}^{2}\right] /\left(S M P_{0}\right)^{2}
\end{aligned}
$$

The first term of both $P(\mathrm{e} / 0)$ and $P(\mathrm{e} / 1)$ corresponds to the error probability that would be obtained if ISI was bounded by the Eye diagram. The second term is the inserted correction in order to consider the random quality of the transmitted digital message. Because the different interfering samples are arranged in absolute value, the computing time is reasonably short.

\subsection{Receiver optimization procedure}

The objective of the receiver optimization procedure is to maximize the repeater spacing. In this paper, for optimum design of an equalized receiver, we follow the strategy proposed in [3].

Once the link distance $L$ is fixed, the object of optimization consists in minimizing the threshold value of the received optical power, $P_{0}(L)$, which is necessary in order to have an error probability 


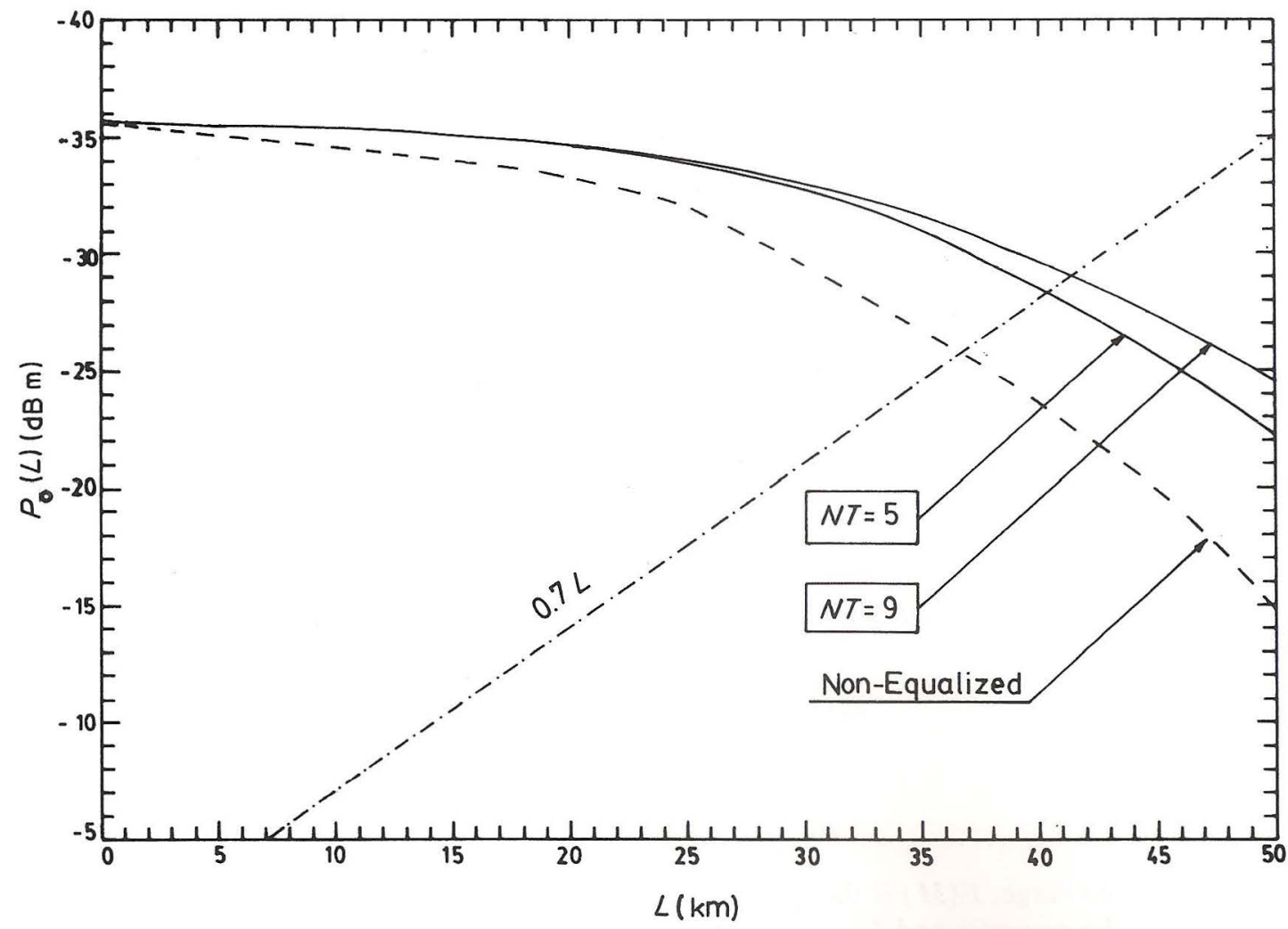

Figure 4 Threshold value of received optical power plotted against repeater spacing.

lower than $10^{-9}$. The inputs to the optimization procedure are the photodetector parameters, $S$, $F(M), I_{\mathrm{M}}$, the thermal-noise power generated in the amplifier, $\sigma_{\mathrm{T}}^{2}$, the low-pass-filter frequency response, $H_{3}(f)$, as a function of the cut-off frequency, $F_{\mathrm{c}}$, the maximum number of equalizer stages, the fibre bandwidth and its attenuation, the bit rate $R$ and the initial values of $P_{0}$ and $F_{\mathrm{c}}$.

The optimization procedure can be outlined in the following steps:

1. Once the low-pass-filter bandwidth, $F_{\mathrm{c}}$, the avalanche gain, $M$, and the receiver optical power, $P_{0}(L)$, are fixed we obtain the equalizer tap coefficients by using Equations 13 to 26 .

2 . Once the values of equalizer coefficients are known, we compute the detector threshold and the bit-error rate (BER). The optimum detector threshold will be obtained when $P(\mathrm{e} / 0)=P(\mathrm{e} / 1)$. We assumed that the data are statistically independent and equiprobable.

3. The minimum values of $P_{0}(L)$ and $M$ which are necessary to have a BER lower than $10^{-9}$ are calculated.

4. The structure, number of stages of upper and lower parts and the optimum values for coefficient taps that minimize $P_{0}(L)$ are obtained.

5. The optimum $F_{\mathrm{c}}$ value to minimize $P_{0}(L)$ is calculated. This optimum value results from a compromise between noise level and ISI.

\section{Results}

We have obtained results from $140 \mathrm{Mbits} \mathrm{s}^{-1}$ and the following parameters:

1. Line code RZ (return-to-zero) with $50 \%$ of duty cycle.

2. Peak power injected in the fibre by the laser diode $P(\mathrm{dBm})=0$. 
TABLE I Comparison between FTSE and CE (both structures compensate one interfering precursor and one interfering postcursor sample)

\begin{tabular}{rll}
\hline$L(\mathrm{~km})$ & $\begin{array}{l}P_{0}(L)(\mathrm{dBm}) \\
\text { for FTSE } \\
\text { (5 stages) }\end{array}$ & $\begin{array}{l}P_{0}(L)(\mathrm{dB} \mathrm{m}) \\
\text { for CE } \\
\text { (3 stages })\end{array}$ \\
\hline 5 & -35.5 & -35.5 \\
10 & -35.3 & -35.2 \\
15 & -35.1 & -34.9 \\
20 & -34.7 & -34.5 \\
25 & -33.8 & -33.6 \\
30 & -32.3 & -32.0 \\
35 & -30.6 & -30.3 \\
40 & -28.8 & -28.4 \\
45 & -25.5 & -25.0 \\
\hline
\end{tabular}

3. Optical-fibre impulse response

$$
h_{2}(t)=\left[\frac{\pi}{\ln 2}\right]^{1 / 2} f_{0}(L) \exp \left[-\left(\pi f_{0}(L) t\right)^{2} / \ln 2\right]
$$

where $f_{0}(L)$ is the cut-off frequency, $-3 \mathrm{~dB}$ at optical power, and it follows a law of type

$$
f_{0}(L)=L^{\gamma} / f_{0 / 1 \mathrm{~km}}
$$

where $f_{0 / 1 \mathrm{~km}}$ is the bandwidth of $1 \mathrm{~km}$ fibre due to the modal dispersion, $\gamma$ is the factor for the dependence of the modal bandwidth on length. Because we have chosen to work at a wavelength of approximately $1.3 \mu \mathrm{m}$, in the above expression the material dispersion has not been considered.

The value of the factor $\gamma$ cannot be anticipated, but it usually ranges between 0.5 and 1 . We have chosen a value of 0.7 . The typical values of the fibre parameters used in the calculations are: $f_{0 / 1 \mathrm{~km}}=800 \mathrm{MHz}$ and $\alpha=0.7 \mathrm{~dB} \mathrm{~km}^{-1}$.

We have considered a germanium photodiode, Optitron GA-1-U, whose characteristic parameters are: $S=0.68 \mathrm{AW}^{-1}$, a photodiode capacity of $0.5 \mathrm{pF}$, and an avalanche dark current of $0.1 \mu \mathrm{A}$.

As the powers used are extremely small, it is very important to optimize the noise level of the amplifier. In order to minimize the noise power generated in the amplifier, optimum bias condition and voltage gain have been chosen. We have assumed that the two first stages have high gain and we can neglect the noise generated in the other stages. The first stage is a shunt feedback preamplifier with bipolar junction transistor (BJT), followed by a BJT with emitter resistor as second stage. In this work, we have chosen the NPN silicon BJT type BFR91, because it has a transition frequency of several gigahertz, a significant gain and a low noise level.

The low-pass filter has been chosen with a gaussian frequency response, whose $-3 \mathrm{~dB}$ bandwidth, $F_{\mathrm{c}}$, must be optimized in order to give a minimum noise level. The low-pass filter response is given by

$$
H_{3}(f)=\exp \left\{-\ln 2\left(f / F_{\mathrm{c}}\right)^{2}\right\}
$$

The numerical results obtained for FTSE are indicated in Fig. 4. Curves stand for the threshold value of received optical power, $\left[P_{0}(L)\right] \mathrm{dB} \mathrm{m}$, as a function of $L$. The intersection of these curves with the straight line $\left[P_{0}(l)\right](\mathrm{dBm})=P(\mathrm{dBm})-\alpha L$ defines the maximal repeater spacing. In particular, we have considered five and nine taps for the equalizer. The results obtained are very similar to the ones obtained in [3] for a conventional $T$-spaced equalizer (CE) with three and five stages, respectively. In Table I we are comparing the results obtained for CE and FTSE, considering that both structures compensate one interfering precursor and one interfering postcursor sample. 


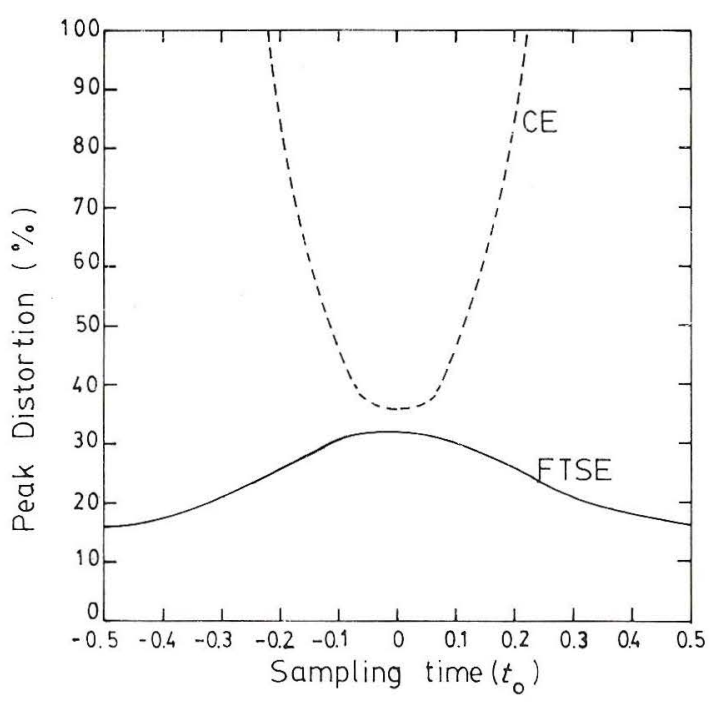

Figure 5 Normalized peak distortion plotted against timing phase for a repeater spacing of $40 \mathrm{~km}$.

In order to obtain comparisons, the figure also shows the results obtained by using an unequalized receiver.

It is also interesting to analyse the influence of the timing offset on the performances of the equalized system. In order to compute the residual amount of ISI on the equalized signal, we define the normalized peak distortion as

$$
D_{\mathrm{p}}(\%)=\frac{1}{h_{\mathrm{E}}\left(t_{0}\right)}\left\{\sum_{\substack{n=-\infty \\ n \neq 0}}^{\infty}\left|h_{\mathrm{E}}\left(t_{0}-n T\right)\right|\right\} \times 100
$$

In particular we have considered $L=40 \mathrm{~km}$. This corresponds approximately to the maximum distance between repeaters that the system can reach, as shown in Fig. 4.

We have plotted in Fig. 5 the values of $D_{\mathrm{p}}$ against the timing offset. The continuous line shows the results obtained for a FTSE with delay spacing, $\tau=T / 2$, and five stages. (This structure can compensate for one precursor and one postcursor $T$-spaced interfering sample.) The dotted line shows the same result for $\mathrm{CE}$ with three stages. The above results show that the dependence of $\mathrm{CE}$ on the timing phase is very sensitive. Really, when the sampling instant is shifted more than $\pm 0.22 T$ the normalized peak distortion becomes greater than one. Then, the Eye diagram closes and the performance of the system drops quickly. However, when FTSE is used, the value of $D_{\mathrm{p}}$ changes only from $15.6 \%$ to $32.3 \%$ when the values of the sampling instant range between $-0.5 T$ and $0.5 T$.

This behaviour of FTSE can be explained as follows. The equalizer taps are designed in order to obtain an equalized impulse response, $h_{\mathrm{E}}(t)$, that accomplishes the Nyquist criterion (in this case no ISI is present). In the frequency domain this condition can be expressed as [13]

$$
\sum_{n} H_{4}\left(f-\frac{n}{T}\right) H\left(f-\frac{n}{T}\right) \exp \left(-j 2 \pi n t_{0} / T\right)=T ; \quad|f| \leqslant 1 / 2 T
$$

where $H_{4}(f)$ and $H(f)$ are the frequency responses of the equalizer and unequalized system, respectively.

When CE is used, $H_{4}(f)$ is a periodic function in $1 / T$. Then $H_{4}[f-(n / T)]=H_{4}(f)$, and Equation 34 becomes

$$
H_{4}(f) \sum_{n} H\left(f-\frac{n}{T}\right) \exp \left(-j 2 \pi n t_{0} / T\right)=T ; \quad|f| \leqslant 1 / 2 T
$$

In this case adequate equalization is not possible for all values of sampling instant, $t_{0}$, because the 


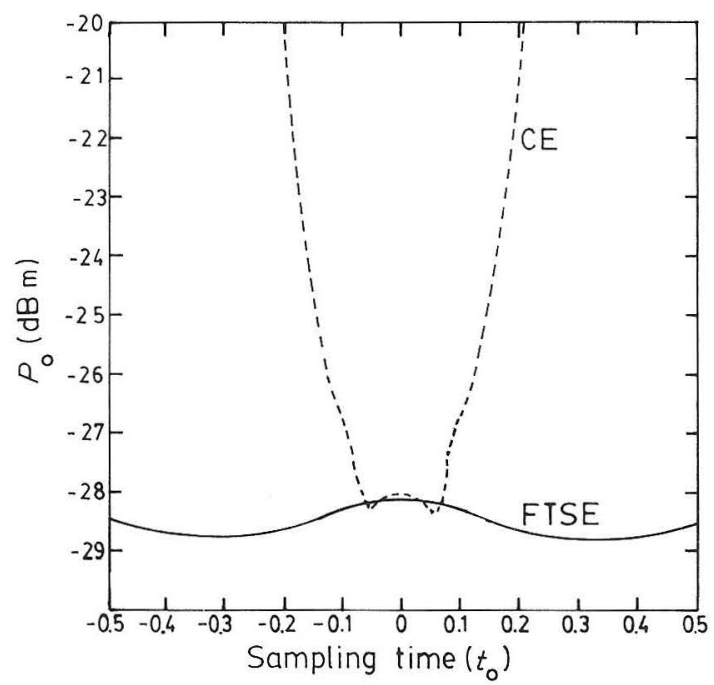

Figure 6 Threshold value of received optical power plotted against timing phase for a repeater spacing of $40 \mathrm{~km}$.

equalizer cannot alter the relative magnitudes and phases of the signal components. These magnitudes and phases are $1 / T \mathrm{~Hz}$ apart in the spectrum and, by the fact of sampling the equalizer output signal at clock rate $(1 / T)$, they become superimposed. For certain values of $t_{0}$, the overlapping of spectral roll-offs of $H(f)$, which are equal in magnitude, becomes opposite in phase and its sum produces cancellation of the overlapping spectral components around $\pm 1 / 2 T$.

For $\tau<T$, the period of $H_{4}(f)$ becomes larger than $1 / T \mathrm{~Hz}$. Hence, in Equation 40, $H_{4}(f-(n / T)]$ cannot be extracted as a common multiplier factor, and by choosing appropriate tap values it will then be always possible to avoid the cancellation of the overlapping spectral components in Equation 40.

From the viewpoint of system performance, it is necessary to analyse the effect of the timing phase on the threshold value of received optical power. In Fig. 6, we have plotted the evolution of $P_{0}(L)$ against the timing phase for both CE and FTSE, and linear structure. For CE, when the sampling instant, $t_{0}$, is shifted $\pm 0.15 T$, the value of $P_{0}(L)$ can be increased $3 \mathrm{~dB}$ in order to maintain the same performance. Moreover, when $t_{0}$ is shifted $\pm 0.22 T$, the value of $P_{0}(L)$ can be increased by $10 \mathrm{~dB}$.

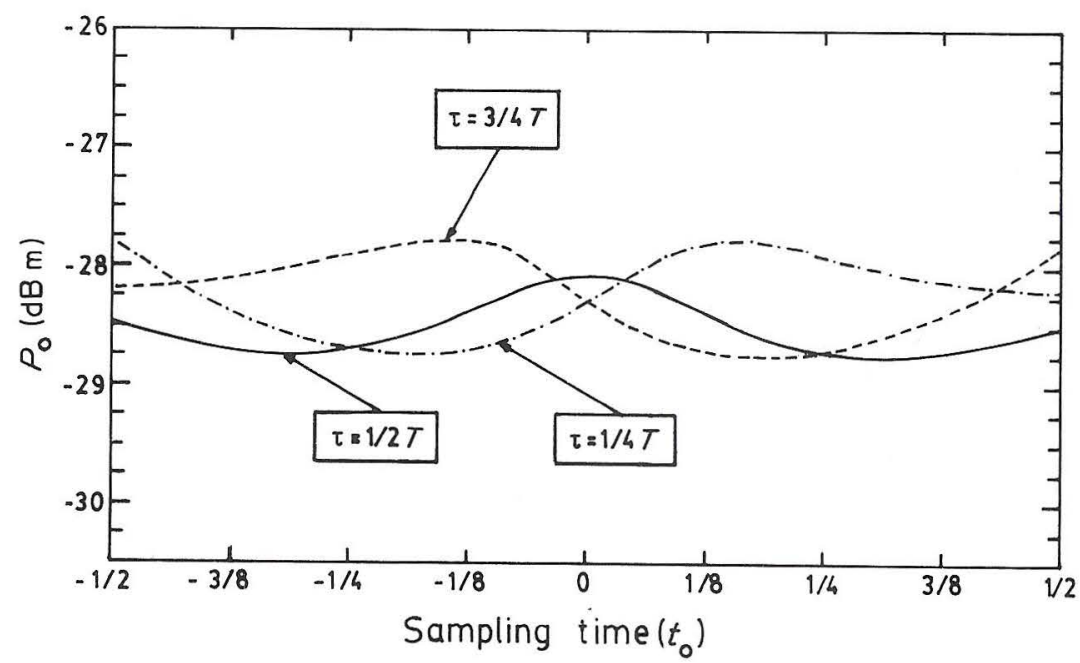

Figure 7 Threshold value of received optical power plotted against timing phase for different values of delay $\tau$. 
In this case, as we have shown above, the system performance really does drop quickly when the sampling instant is shifted.

When FTSE is considered, the value of the threshold of received optical power only varies $0.5 \mathrm{~dB}$ for sampling instants ranging between $-0.5 T$ and $0.5 T$. Then the system performance is very insensitive to timing phase.

Fig. 7 also shows the influence of the delay $\tau$ in the system performance. Values of $\tau=1 / 4 T$, $\tau=1 / 2 T, \tau=3 / 4 T$ and $L=40 \mathrm{~km}$ have been chosen. For all of them the threshold value of received optical power ranges between -28.7 and $-27.8 \mathrm{dBm}$. Then the maximum variation is lower than $1 \mathrm{~dB}$.

The results obtained indicate that when the structure shown in Fig. 3 is used to construct a FTSE the equalizer results are almost insensitive to the value of the delay $\tau$ between the upper and lower parts.

\section{Conclusions}

The results obtained show that the fractional tap-spacing equalizer is very insensitive to the timing phase for all the structures analysed. For the linear structure and $L=40 \mathrm{~km}$ the received optical power threshold changes only from -28.1 to $-28.7 \mathrm{dBm}$ when the sampling instant ranges between $-0.5 T$ and $0.5 T$. This represents a change of only $2.13 \%$ on the threshold value of the optical power.

The insensitivity to the timing phase allows the use of a simple clock recovery scheme. Furthermore, if a LMS algorithm is used to equalize the unknown channels automatically, the insensitivity to the timing phase makes the algorithm independent of the clock phase.

\section{Appendix}

\section{Autocorrelation function of $x(t)$}

Because of optical power and/or thermal excitation, primary electron-hole pairs are generated at various points within the photodetector. The number of primary pairs generated at any time interval can be considered statistically independent of pairs generated at any other disjoint interval. The generation of primary carriers can therefore be described by Poisson statistics with intensity $\lambda(t)$ [2].

We divide the time axis into very small intervals of width $\Delta t$ infinitesimal, and call $n_{m}$ the pairs generated at the $m$ th interval [14], then

$$
\begin{aligned}
& E\left(n_{m}\right) \cong \lambda\left(t_{m}\right) \Delta t \\
& E\left(n_{m}^{2}\right) \cong \lambda\left(t_{m}\right) \Delta t
\end{aligned}
$$

When primary carriers pass through the high-field region, they may gain sufficient energy to generate other electron-hole pairs through collision ionization. These new pairs can, in turn, generate additional pairs by the same mechanism. Then, the current leaving the photodetector consists of 'bunches' of electrons and the filter output signal can be formulated as

$$
x(t)=x_{1}(t)+n_{\mathrm{T}}(t)
$$

where

$$
x_{1}(t) \cong q \sum_{m=-\infty}^{\infty} n_{m} g_{m} h_{3}\left(t-t_{m}\right)
$$

$n_{\mathrm{T}}(t)$ is the thermal noise and $g_{m}$ is the avalanche ionization gain in the $m$ th interval.

The autocorrelation function of $x_{1}(t)$ is

$$
\begin{aligned}
R_{x_{1} x_{1}}\left(t_{1}, t_{2}\right) & =E\left[x\left(t_{1}\right) x\left(t_{2}\right)\right] \\
& \cong q^{2} E\left[\sum_{m} n_{m} g_{m} h_{3}\left(t_{1}-t_{m}\right) \sum_{l} n_{l} g_{l} h_{3}\left(t_{2}-t_{l}\right)\right]
\end{aligned}
$$




$$
\begin{aligned}
= & q^{2} \sum_{\substack{m \\
m \neq l}} \sum_{l} E\left(n_{m} n_{l} g_{m} g_{l}\right) h_{3}\left(t_{1}-t_{m}\right) h_{3}\left(t_{2}-t_{l}\right) \\
& +q^{2} \sum_{m} E\left(n_{m}^{2} g_{m}^{2}\right) h_{3}\left(t_{1}-t_{m}\right) h_{3}\left(t_{2}-t_{m}\right)
\end{aligned}
$$

Under the assumption that the electric field along the photodetector is independent of the number of carriers generated, we can state that all ionizing collisions are statistically independent. From Equation Al we obtain

$$
E\left(n_{m} n_{l} g_{m} g_{l}\right) \cong \begin{cases}M_{1}^{2} \lambda\left(t_{m}\right) \lambda\left(t_{l}\right) \Delta t^{2} ; & m \neq l \\ M_{2} \lambda\left(t_{m}\right) \Delta t ; & m=l\end{cases}
$$

where $M_{1}$ and $M_{2}$ are the first and second moments of the random variable avalanche gain, whose general expression is given by [15]

$$
M_{n}=M_{n-1} M_{1}+\left(1-M_{1}\right) \sum_{m=1}^{n-1}\left(\begin{array}{c}
n-1 \\
m
\end{array}\right) M_{m}\left[(K-1) M_{n-m-1}-K M_{n-m}\right] ; \quad n \geqq 2
$$

with $M_{1}=M$ and $K$ a parameter which depends on the material and doping profile of the avalanche diode.

From Equations A5 and A6, we obtain

$$
\begin{aligned}
R_{x_{1} \cdot x_{1}}\left(t_{1}, t_{2}\right) \cong & q^{2} M_{1}^{2} \sum_{\substack{m \\
m \neq i}} \sum_{l} \lambda\left(t_{m}\right) h_{3}\left(t_{1}-t_{m}\right) \Delta t \lambda\left(t_{1}\right) h_{3}\left(t_{2}-t_{l}\right) \Delta t \\
& +q^{2} M_{2} \sum_{m} \lambda\left(t_{m}\right) h_{3}\left(t_{1}-t_{m}\right) h_{3}\left(t_{2}-t_{m}\right) \Delta t
\end{aligned}
$$

If $\Delta t \rightarrow 0$, the autocorrelation function is

$$
\begin{aligned}
R_{x_{1} x_{1}}\left(t_{1}, t_{2}\right)= & q^{2} M_{1}^{2}\left[\lambda\left(t_{1}\right) * h_{3}\left(t_{1}\right)\right]\left[\lambda\left(t_{2}\right) * h_{3}\left(t_{2}\right)\right] \\
& +q^{2} M_{2} \lambda\left(t_{2}\right) h_{3}\left(t_{1}-t_{2}\right) * h_{3}\left(t_{2}\right)
\end{aligned}
$$

Finally, we have

$$
R_{x x}\left(t_{1}, t_{2}\right)=R_{x_{1} x_{1}}\left(t_{1}, t_{2}\right)+R_{N N}\left(t_{1}, t_{2}\right)
$$

where $R_{N N}\left(t_{1}, t_{2}\right)$ is the autocorrelation function of $n_{\mathrm{T}}(t)$.

\section{References}

1. D. G. MESSERSCHMitT, IEEE Trans. Commun. COM26 (1978) 1110.

2. B. L. KASPER, Bell Syst. Tech. J. 61 (1982) 1367.

3. F. CASADEVAll, G. JUNYENT and R. AGUSti, Opt. Quantum Electron. 16 (1984) 377.

4. R. A. RUGEMAliRA, IEEE Trans. Commun. COM33 (1985) 158.

5. D. F. FALCONER and J. SALZ, IEEE Trans. Inform. Theory IT23 (1977) 117.

6. H. KOBAYASHI, IEEE Trans. Commun. COM19 (1971) 268.

7. E. ROSA, ibid. COM22 (1974) 1236.

8. G. UNGERBOECK, ibid. COM24 (1976) 856.

9. J. SALZ, ibid. COM25 (1977) 1471.

10. C. VASAllo, IEEE Trans. Microwave Theory MIT-25 (1977) 572.

11. R. DOGLOTti, A. GUARDinCERI, A. LUVISON and G. PIRANi, CSELT Rapp. Tec. 3 (1976) 141.

12. L. GUIDOUX and O. MACCHI, Ann. Telecomm. 55 (1975) 331.

13. R. W. LUCKY, J. SALZE. D. WELDON, 'Principles of Data Communication' (McGraw-Hill, New York, 1968).

14. V. OSTOICH, 'PCM Optical Fibre Communications Systems' (Technical University, Denmark, 1977).

15. W. HAUK, F. BROSS and M. OTIKA, IEEE Trans. Commun. COM26 (1978) 1119. 